\title{
A Criterion for Essential Self-Adjointness of a Symmetric Operator Defined by Some Infinite Hermitian Matrix with Unbounded Entries
}

\author{
Tomasz Komorowski
}

\begin{abstract}
We shall consider a double infinite, Hermitian, complex entry matrix $A=\left[a_{x, y}\right]_{x, y \in \mathbb{Z}}$. In the present note we give a criterion, expressed in terms of the entries of the matrix, for the corresponding symmetric operator defined on compactly supported sequences, to be essentially self-adjoint in the space $\ell_{2}(\mathbb{Z})$. Roughly speaking, assuming that $x$ denotes the row number, we require that: (1) there exist $\gamma \in[0,1)$ and $n>0$ for which the entries that are at distance larger than $n\left(|x|^{2}+1\right)^{\gamma / 2}$ from the diagonal vanish and (2) the $\ell^{1}$ norm of the $x$ th row grows slower that $|x|^{\gamma-1}$, as $|x| \rightarrow+\infty$.
\end{abstract}

Keywords. Hermitian matrix, self-adjoint operator.

\section{Introduction}

We shall consider a double infinite, Hermitian, complex entry matrix $A=$ $\left[a_{x, y}\right]_{x, y \in \mathbb{Z}}$, with $a_{x, y}^{*}=a_{y, x}, x, y \in \mathbb{Z}$. Here $a^{*}$ denotes the complex conjugate of $a \in \mathbb{C}$. We assume furthermore that the matrix is almost of a finite bandwidth, i.e. there exists an integer $n \geq 1$ and exponent $\gamma \in[0,1)$ such that

$$
a_{x, x+z}=0, \quad z>n\langle x\rangle^{\gamma}, \quad x \in \mathbb{Z} .
$$

Here, for given $a$ we let $\langle a\rangle:=\left(1+|a|^{2}\right)^{1 / 2}$. With the help of the matrix $A$ we can define a symmetric operator on the subset $c_{0}(\mathbb{Z})$ of the complex Hilbert space $\ell_{2}(\mathbb{Z})$ - the space consisting of all double infinite sequences $f=\left(f_{x}\right)$ equipped with the norm

$$
\|f\|_{\ell_{2}(\mathbb{Z})}:=\left\{\sum_{x}\left|f_{x}\right|^{2}\right\}^{1 / 2}<+\infty .
$$

\footnotetext{
The author acknowledges the support of the Polish National Science Center grant DEC-
} 2012/07/B/SR1/03320. 
Here $c_{0}(\mathbb{Z})$ is the subspace containing all compactly supported $f$. The operator is given by

$$
(A f)_{x}:=\sum_{y} a_{x y} f_{y}, \quad x \in \mathbb{Z}, \quad f \in c_{0}(\mathbb{Z}) .
$$

According to Theorem 4, p. 102 of [1], assumption (1.1) implies that the operator is closable. Denote its closure by $\bar{A}: D(\bar{A}) \rightarrow \ell_{2}(\mathbb{Z})$. In our principal result, see Theorem 2.1 below, we formulate a sufficient condition, in terms of the growth of $\left|a_{x y}\right|$, see (2.4) below, for the operator $\bar{A}$ to be self-adjoint. The above means that the index of deficiency of $A: c_{0}(\mathbb{Z}) \rightarrow \ell_{2}(\mathbb{Z})$ equals $(0,0)$, see [4], Theorem V.3.16, p. 271.

Note that in the particular case when $\gamma=0$ the assumption (1.1) is equivalent with the existence of $n$ such that

$$
a_{x, y}=0, \quad|x-y|>n
$$

and the definition coincides with the usual definition of $(n J)$-matrices, see [14], (sometimes also called finite bandwidth matrices). When $n=1$ they are called Jacobi matrices and play an important role in the theory of the Hamburger moment problem. This case has been well studied in the literature, see e.g. $[6-9,11,13]$ and the references contained therein, although also then our results formulated in Corollary 2.2 and Theorem 2.3 below also seem to be new. The case of a symmetric matrix with possibly unbounded coefficients and possessing a barrier function has been studied in [3], see Theorem 2.3 of ibid. The sufficient conditions for self-adjointness given in [3] seem to be quite different from those stated in our main results, see Sect. 2 below.

\section{The Statement of the Main Result}

Since the matrix $A=\left[a_{x, y}\right]$ is Hermitian the operator $\bar{A}$ is obviously symmetric, i.e.

$$
\langle g, \bar{A} f\rangle_{\ell_{2}(\mathbb{Z})}=\langle\bar{A} g, f\rangle_{\ell_{2}(\mathbb{Z})}, \quad f, g \in D(\bar{A}) .
$$

Here, $\langle\cdot, \cdot\rangle_{\ell_{2}(\mathbb{Z})}$ denotes the usual scalar product in $\ell_{2}(\mathbb{Z})$. Let $f \in \ell_{2}(\mathbb{Z})$ be such that the functional

$$
\varphi(g):=\langle\bar{A} g, f\rangle_{\ell_{2}(\mathbb{Z})}, \quad g \in D(\bar{A})
$$

is bounded, i.e. for some $C>0$

$$
|\varphi(g)| \leq C\|g\|_{\ell_{2}}, \quad g \in D(\bar{A}) .
$$

Self-adjointness of $\bar{A}$ means that any $f$, for which (2.3) holds, belongs to $D(\bar{A})$ and, as a consequence $(2.1)$ is in force.

For example, if there exists $M>0$ such that $\sum_{y}\left|a_{x y}\right| \leq M$ for all $x \in \mathbb{Z}$ then $\bar{A}$ is bounded on $\ell_{2}(\mathbb{Z})$, see Example III.2.3, p. 143 of [4], therefore it is self-adjoint. Our main result can be stated as follows. 
Theorem 2.1. Suppose that for some $\gamma \in[0,1)$ the entries of matrix A satisfy both condition (1.1) and

$$
\lim _{|x| \rightarrow+\infty} \frac{1}{\langle x\rangle^{1-\gamma}}\left(\sum_{y}\left|a_{x y}\right|\right)=0 .
$$

Then, the operator $A$, given by (1.2), is essentially selfadjoint on $\ell_{2}(\mathbb{Z})$.

Using the theorem for $\gamma=0$ we immediately conclude the following.

Corollary 2.2. The conclusion of Theorem 2.1 holds when $A$ is a Hermitian, $n J$-matrix (i.e. (1.3) is in force) whose entries satisfy

$$
\lim _{|x| \rightarrow+\infty} \frac{1}{\langle x\rangle}\left(\sum_{y}\left|a_{x y}\right|\right)=0 .
$$

In fact, in the case of $n J$-matrices, one can show a little stronger result, relaxing a bit assumption (2.5).

Theorem 2.3. Suppose that condition (1.3) holds. Then, there exists $c_{*}>0$ depending only on $n$ such that the conclusion of Theorem 2.1 holds for any Hermitian $n J$-matrix $A=\left[a_{x y}\right]$ that satisfies

$$
\limsup _{|x| \rightarrow+\infty} \frac{1}{\langle x\rangle}\left(\sum_{y}\left|a_{x y}\right|\right) \leq c_{*} .
$$

Example. The condition (2.6) is in some sense optimal. Suppose that $\delta>1$ is arbitrary. Consider the Jacobi matrix with entries given by

$$
a_{x, x+z}= \begin{cases}0, & \text { if } x \leq 0, \quad \text { or } \quad x+z \leq 0, \text { or } z=0, \text { or } z>2, \\ x^{\delta}, & \text { if } x>0 \text { and } z=1\end{cases}
$$

According to Corollary 1, p. 267 of [6] the index of deficiency of the respective operator $A: c_{0}(\mathbb{Z}) \rightarrow \ell_{2}(\mathbb{Z})$ equals then (1.1). Therefore $A$ cannot be essentially self-adjoint, see Corollary 2.2 of [13].

\section{Proof of Theorem 2.1}

Recall the classical criterion for the essential self-adjointness of a symmetric operator, see Theorem 3 of Section 33.2 of [10], or Corollary 2.2 of [13]. Adjusted to our settings it reads as follows: suppose that a closed operator $\bar{A}$ is symmetric and

$$
R(I-i \bar{A})=\ell_{2}(\mathbb{Z})=R(I+i \bar{A}) .
$$

Then, it is self-adjoint.

To prove (3.1) we show the following result.

Proposition 3.1. Under the assumptions of Theorem 2.1 for any $g=\left(g_{x}\right) \in$ $c_{0}(\mathbb{Z})$ there exists $f=\left(f_{x}\right)$ such that

$$
f_{x}-i \sum_{y} a_{x y} f_{y}=g_{x}, \quad x \in \mathbb{Z}
$$


and

$$
\sum_{x}\langle x\rangle^{2 k}\left|f_{x}\right|^{2}<+\infty, \quad k=1,2, \ldots
$$

Observe that the infinite summation range appearing in equation (3.2) is in fact finite. Indeed, from (1.1) and the symmetry it follows that

$$
a_{x, x-z}=0, \quad z>c_{n}\langle x\rangle^{\gamma}
$$

where

$$
c_{n}:=\max \left\{2 n, 2^{(1+\gamma / 2) /(1-\gamma)} n^{1 /(1-\gamma)}\right\} .
$$

Combining this with (1.1) we conclude that

$$
a_{x, x+z}=0, \quad|z|>c_{n}\langle x\rangle^{\gamma} .
$$

Before showing Proposition 3.1 we use it to prove the equalities appearing in (3.1), finishing in this way the proof of Theorem 2.1. Consider $f_{x}^{(N)}:=f_{x} 1_{[|x| \leq N]}$. We claim that

$$
(I-i A) f^{(N)} \rightarrow g \text { and } \quad f^{(N)} \rightarrow f
$$

strongly in $\ell_{2}(\mathbb{Z})$, as $N \rightarrow+\infty$. The latter convergence is obvious. We only show how to prove the first claim of (3.7). Note that

$$
(I-i A) f_{x}^{(N)}=f_{x}-i \sum_{y} a_{x y} f_{y}+R_{x}^{(N)}+r_{x}^{(N)}=g_{x}+R_{x}^{(N)}+r_{x}^{(N)},
$$

where

$$
r_{x}^{(N)}:=f_{x}^{(N)}-f_{x}, \quad R_{x}^{(N)}:=i \sum_{|y|>N} a_{x y} f_{y} .
$$

It suffices to prove that

$$
\lim _{N \rightarrow+\infty}\left\|R^{(N)}\right\|_{\ell^{2}}=0
$$

Observe that there exists a constant $\tilde{c} \in(0,1)$, depending only on $c_{n}$ and $\gamma \in[0,1)$ such that

$$
\tilde{c}\langle x\rangle \leq\langle y\rangle \leq \frac{\langle x\rangle}{\tilde{c}}, \quad x \in \mathbb{Z},|y-x| \leq c_{n}\langle x\rangle^{\gamma} .
$$

In particular, if $|y| \geq N$ and $|y-x| \leq c_{n}\langle x\rangle^{\gamma}$ then $\langle x\rangle \geq \tilde{c} N$. Therefore

$$
R_{x}^{(N)}=0, \quad\langle x\rangle<\tilde{c} N
$$

On the other hand, for $\langle x\rangle \geq \tilde{c} N$ we have

$$
\left|R_{x}^{(N)}\right| \leq \sum_{|y-x| \leq c_{n}\langle x\rangle^{\gamma}}\left|a_{x y}\right|\left|f_{y}\right| \leq \max _{y}\left|a_{x y}\right| \sum_{|y-x| \leq c_{n}\langle x\rangle^{\gamma}}\left|f_{y}\right| .
$$


Using Cauchy-Schwartz inequality and (3.3) we conclude that for any $k>2$ there exists $C>0$ such that

$$
\begin{aligned}
\sum_{|y-x| \leq c_{n}\langle x\rangle^{\gamma}}\left|f_{y}\right| & =\sum_{|y-x| \leq c_{n}\langle x\rangle^{\gamma}}\langle y\rangle^{-k}\langle y\rangle^{k}\left|f_{y}\right| \\
& \leq\left\{\sum_{|y-x| \leq c_{n}\langle x\rangle^{\gamma}}\langle y\rangle^{-2 k}\right\}^{1 / 2}\left\{\sum_{|y-x| \leq c_{n}\langle x\rangle^{\gamma}}\langle y\rangle^{2 k}\left|f_{y}\right|^{2}\right\}^{1 / 2} \\
& \leq \frac{C}{\langle x\rangle^{k-1 / 2}}
\end{aligned}
$$

for all $x \in \mathbb{Z}$. From (2.4) we get that for some $C>0$

$$
\max _{y}\left|a_{x y}\right| \leq C\langle x\rangle^{1-\gamma}, \quad x \in \mathbb{Z} .
$$

Using (3.13) and (3.14) to bound the right hand side of (3.12) we get

$$
\left|R_{x}^{(N)}\right| \leq \frac{C}{\langle x\rangle^{k-3 / 2+\gamma}}, \quad\langle x\rangle \geq \tilde{c} N .
$$

for some constant $C>0$. Combing this with (3.11) we conclude (3.9), thus finishing the proof of (3.7).

From (3.7) we conclude that any $f$ satisfying both (3.2) and (3.3) belongs to $D(\bar{A})$. Indeed, since each $f^{(N)}$ belongs to $D(A)$ (as it is compactly supported) and $\bar{A}$ is the closure of $A$, we infer from (3.7) that $f \in D(\bar{A})$ and $(I-i \bar{A}) f=g$. Due to the fact that $c_{0}(\mathbb{Z})$ is dense in $\ell_{2}(\mathbb{Z})$ and that the range $R(I-i \bar{A})$ is closed we obtain that $R(I-i \bar{A})=\ell_{2}(\mathbb{Z})$. The proof of the second equality in (3.1) goes along the same lines. The only thing that remains yet to be shown is therefore Proposition 3.1.

Proof of Proposition 3.1. Let

$$
\chi_{N}(r):= \begin{cases}r, & |r| \leq N, \\ N, & r \geq N, \\ -N, & r \leq-N .\end{cases}
$$

For a fixed integer $N$ define $A^{(N)}$ as a bounded, symmetric operator corresponding to the Hermitian matrix whose entries equal

$$
a_{x y}^{(N)}:=\chi_{N}\left(a_{x y}\right) 1_{[|x-y| \leq N]}, \quad x, y \in \mathbb{Z} .
$$

Given $g \in c_{0}(\mathbb{Z})$ there is a (unique) $\tilde{f}^{(N)} \in \ell_{2}(\mathbb{Z})$ such that

$$
\left(I-i A^{(N)}\right) \tilde{f}^{(N)}=g .
$$

We show that for any positive integer $k$ there exists a constant $C>0$ such that

$$
\sum_{x}\langle x\rangle^{2 k}\left|\tilde{f}_{x}^{(N)}\right|^{2} \leq C, \quad N \geq 1 .
$$

Taking this claim for granted (its proof shall be shown momentarily) we finish the proof of the proposition. Using condition (3.17) with any $k>0$ we conclude that the tails of the infinite sums defining the $\ell_{2}(\mathbb{Z})$ norms of $\left(\tilde{f}^{(N)}\right)$ are uniformly small in $N$. This proves that the sequence is strongly 
precompact in $\ell_{2}(\mathbb{Z})$, see e.g. Theorem 4.20 .1 of [2]. In fact, observe that each $\tilde{f}^{(N)} \in D(\bar{A})$. Indeed, let $\tilde{f}^{(N, M)}:=\left(\tilde{f}_{x}^{(N)} 1_{[|x| \leq M]}\right)$ for an integer $M \geq 1$. Of course $\tilde{f}^{(N, M)} \in c_{0}(\mathbb{Z}) \subset D(\bar{A})$, and it converges to $\tilde{f}^{(N)}$ strongly in $\ell_{2}(\mathbb{Z})$, as $M \rightarrow+\infty$. On the other hand, from (3.17) for any $k, \tilde{c}>0$ there exists $C>0$ such that

$$
\sup _{\langle y\rangle \geq \tilde{c}\langle x\rangle}\left|\tilde{f}_{y}^{(N)}\right|^{2} \leq \frac{C}{\langle x\rangle^{2 k+4}}, \quad N \geq 1, x \in \mathbb{Z} .
$$

Using (3.6), we can estimate

$$
\begin{aligned}
\sum_{x}\langle x\rangle^{2 k}\left|\left(A \tilde{f}^{(N, M)}\right)_{x}\right|^{2} & \leq \sum_{x}\langle x\rangle^{2 k}\left(\sum_{|y-x| \leq c_{n}\langle x\rangle^{\gamma}}\left|a_{x y}\right|\left|\tilde{f}_{y}^{(N)}\right|\right)^{2} \\
& \leq \sum_{x}\langle x\rangle^{2 k} \sup _{|y-x| \leq c_{n}\langle x\rangle^{\gamma}}\left|\tilde{f}_{y}^{(N)}\right|^{2}\left(\sum_{y}\left|a_{x y}\right|\right)^{2}
\end{aligned}
$$

Since $\gamma \in[0,1)$ there exists $\tilde{c}>0$ such that $\langle y\rangle \geq \tilde{c}\langle x\rangle$ for all $x, y \in \mathbb{Z}$ satisfying the condition $|y-x| \leq c_{n}\langle x\rangle^{\gamma}$, see (3.10). Thanks to (3.18) the utmost right hand side of (3.19) can be estimated then by

$$
\sum_{x}\langle x\rangle^{2 k} \sup _{|y| \geq \tilde{c}|x|}\left|\tilde{f}_{y}^{(N)}\right|^{2}\left(\sum_{y}\left|a_{x y}\right|\right)^{2} \leq C \sum_{x}\langle x\rangle^{-4}\left(\sum_{y}\left|a_{x y}\right|\right)^{2} .
$$

Using the above together with (2.4) we conclude that there exists a constant $C_{1}$ such that

$$
\sum_{x}\langle x\rangle^{2 k}\left|\left(A \tilde{f}^{(N, M)}\right)_{x}\right|^{2} \leq C_{1} \sum_{x}\langle x\rangle^{-2-2 \gamma}, \quad N, M \geq 1 .
$$

In consequence $\left(A \tilde{f}^{(N, M)}\right), M \geq 1$ is strongly precompact in $\ell_{2}(\mathbb{Z})$, for a fixed $N$, and since $\bar{A}$ is the closure of $A$ we obtain $\tilde{f}^{(N)} \in D(\bar{A})$ and

$$
\bar{A} \tilde{f}^{(N)}=\lim _{M \rightarrow+\infty} A \tilde{f}^{(N, M)} .
$$

In addition, we also infer that

$$
\left(\bar{A} \tilde{f}^{(N)}\right)_{x}=\sum_{y} a_{x y} \tilde{f}_{y}^{(N)}, \quad x \in \mathbb{Z}
$$

and that for any $k>0$ there exists a constant $C>0$

$$
\sum_{x}\langle x\rangle^{2 k}\left|\left(\bar{A} \tilde{f}^{(N)}\right)_{x}\right|^{2} \leq C, \quad N \geq 1
$$

From (3.17) and (3.23) we conclude that both sequences $\left(\tilde{f}^{(N)}\right)$ and $\left(\bar{A} \tilde{f}^{(N)}\right)$ are strongly precompact in $\ell_{2}(\mathbb{Z})$. Choosing suitable subsequences, if necessary, we can assume with no loss of generality that $\tilde{f}^{(N)} \rightarrow f$ and $\bar{A} \tilde{f}^{(N)}$ converges to some $h$, strongly in $\ell_{2}(\mathbb{Z})$, as $N \rightarrow+\infty$. Then $f \in D(\bar{A})$ and $\bar{A} f=h$. Estimate (3.3) is a direct consequence of (3.17). In the light of (3.16), 
to finish the proof of (3.2) (thus concluding the proof of the proposition) it suffices to show that

$$
\lim _{N \rightarrow+\infty}\left\|A^{(N)} \tilde{f}^{(N)}-\bar{A} \tilde{f}^{(N)}\right\|_{\ell_{2}(\mathbb{Z})}=0 .
$$

Estimating as in (3.19) and (3.20) we obtain

$$
\begin{aligned}
& \sum_{x}\left|\left(\left(\bar{A}-A^{(N)}\right) \tilde{f}^{(N)}\right)_{x}\right|^{2} \\
& \leq \sum_{x} \sup _{|y| \geq \tilde{c}|x|}\left|\tilde{f}_{y}^{(N)}\right|^{2}\left(\sum_{y}\left|a_{x y}-a_{x y}^{(N)}\right|\right)^{2} \\
& \leq C \sum_{x}\langle x\rangle^{-2 k-4}\left(\sum_{y}\left|a_{x y}-a_{x y}^{(N)}\right|\right)^{2} \rightarrow 0,
\end{aligned}
$$

as $N \rightarrow+\infty$. The passage to the limit on the utmost right hand side can be argued easily by virtue of the Lebesgue dominated convergence theorem. This ends the proof of (3.2), modulo the fact that estimate (3.17) still requires to be shown. Its proof is an adaptation to the present case of an argument used in [12], see also Section 2.7.4 of [5]. Define a bounded operator $T: \ell_{2}(\mathbb{Z}) \rightarrow \ell_{2}(\mathbb{Z})$ by the formula $T f_{x}:=t_{x} f_{x}$, where

$$
t_{x}:=\langle X\rangle^{k} 1_{[|x|<X]}+\langle x\rangle^{k} 1_{[X \leq|x| \leq Y]}+\langle Y\rangle^{k} 1_{[Y<|x|]}, \quad x \in \mathbb{Z}
$$

and $0<X<Y$ are some constants to be determined later on. Directly from (3.25) it follows that

$$
\left|t_{x}-t_{y}\right| \leq\left|\langle x\rangle^{k}-\langle y\rangle^{k}\right|, \quad x, y \in \mathbb{Z} .
$$

Applying $T$ to both sides of (3.16) and taking the inner product against $T \tilde{f}^{(N)}$ on both sides of the aforementioned equation we conclude that

$$
\begin{aligned}
& \left\|T \tilde{f}^{(N)}\right\|_{\ell_{2}(\mathbb{Z})}^{2}+i\left\langle T \tilde{f}^{(N)},\left[T, A^{(N)}\right] \tilde{f}^{(N)}\right\rangle_{\ell_{2}(\mathbb{Z})} \\
& \quad+i\left\langle T \tilde{f}^{(N)}, A^{(N)} T \tilde{f}^{(N)}\right\rangle_{\ell_{2}(\mathbb{Z})}=\left\langle T \tilde{f}^{(N)}, T g\right\rangle_{\ell_{2}(\mathbb{Z}),}
\end{aligned}
$$

where $\left[T, A^{(N)}\right]:=T A^{(N)}-A^{(N)} T$ is the commutator of $T$ and $A^{(N)}$. Thanks to the symmetry of $A^{(N)}$ we have

$$
\operatorname{Re} i\left\langle T \tilde{f}^{(N)}, A^{(N)} T \tilde{f}^{(N)}\right\rangle_{\ell_{2}(\mathbb{Z})}=0 .
$$

Here $\operatorname{Re} z$ and $\operatorname{Im} z$ denote the real and imaginary parts of a complex number $z$. Taking the real part of the expressions appearing on both sides of (3.27) we obtain

$$
\left\|T \tilde{f}^{(N)}\right\|_{\ell_{2}(\mathbb{Z})}^{2}-\operatorname{Im}\left\langle T \tilde{f}^{(N)},\left[T, A^{(N)}\right] \tilde{f}^{(N)}\right\rangle_{\ell_{2}(\mathbb{Z})}=\operatorname{Re}\left\langle T \tilde{f}^{(N)}, T g\right\rangle_{\ell_{2}(\mathbb{Z})} .
$$

Note that

$$
\left[T, A^{(N)}\right] \tilde{f}_{x}^{(N)}=\sum_{y} a_{x y}^{(N)}\left(t_{x}-t_{y}\right) f_{y}^{(N)}
$$

therefore

$$
\left\langle T \tilde{f}^{(N)},\left[T, A^{(N)}\right] \tilde{f}^{(N)}\right\rangle_{\ell_{2}(\mathbb{Z})}=\sum_{x} \sum_{y} a_{y x}^{(N)}\left(t_{x}-t_{y}\right) t_{x} \tilde{f}_{x}^{(N)}\left(\tilde{f}_{y}^{(N)}\right)^{*} .
$$


The above expression can be bounded as follows

$$
\left|\left\langle T \tilde{f}^{(N)},\left[T, A^{(N)}\right] \tilde{f}^{(N)}\right\rangle_{\ell_{2}(\mathbb{Z})}\right| \leq \sum_{x} \sum_{y}\left|a_{x y}\right| t_{x}\left|t_{y}-t_{x}\right|\left|\tilde{f}_{x}^{(N)}\right|\left|\tilde{f}_{y}^{(N)}\right| .
$$

Applying Young's inequality we can estimate the right hand side of (3.29) by $I_{1}+I_{2}$, where

$$
\begin{aligned}
& I_{1}:=\frac{1}{2} \sum_{x} \sum_{y}\left|a_{x y}\right| t_{x}\left|t_{y}-t_{x}\right|\left|\tilde{f}_{x}^{(N)}\right|^{2}, \\
& I_{2}:=\frac{1}{2} \sum_{y} \sum_{x}\left|a_{x y}\right| t_{x}\left|t_{y}-t_{x}\right|\left|\tilde{f}_{y}^{(N)}\right|^{2} .
\end{aligned}
$$

Using condition (3.6) and the fact that $t_{x}$ is constant for $|x| \leq X$, or $Y \leq|x|$ we conclude that

$$
I_{1} \leq \frac{1}{2} \sum_{x=\bar{X}}^{\bar{Y}} \sum_{y=x-c_{n}\langle x\rangle^{\gamma}}^{x+c_{n}\langle x\rangle^{\gamma}}\left|a_{x y}\right| t_{x}\left|t_{x}-t_{y}\right|\left|\tilde{f}_{x}^{(N)}\right|^{2},
$$

where $\bar{X}:=X-c_{n}\langle X\rangle^{\gamma}$ and $\bar{Y}:=Y+c_{n}\langle Y\rangle^{\gamma}$. Thanks to (3.26) we can estimate

$$
I_{1} \leq \frac{1}{2} \sum_{x=\bar{X}}^{\bar{Y}} t_{x}^{2}\left|\tilde{f}_{x}^{(N)}\right|^{2}\left\{\frac{\langle x\rangle^{k}}{t_{x}} \sum_{y=x-c_{n}\langle x\rangle^{\gamma}}^{x+c_{n}\langle x\rangle^{\gamma}}\left|a_{x y}\right|\left|\frac{\langle y\rangle^{k}}{\langle x\rangle^{k}}-1\right|\right\} .
$$

Observe that

$$
\frac{\langle x\rangle^{k}}{t_{x}} \leq\left(c_{n}+1\right)^{k}, \quad \bar{X} \leq x \leq \bar{Y} .
$$

Choose an arbitrary $\delta \in(0,1)$. Note that for $|m| \leq n\langle x\rangle^{\gamma}$ there exist constants $C, C^{\prime}>0$ such that

$$
\left|\left(\frac{1+(x+m)^{2}}{1+x^{2}}\right)^{k / 2}-1\right| \leq C \frac{|x m|+m^{2} / 2}{1+x^{2}} \leq C^{\prime}\left(\langle x\rangle^{\gamma-1}+\langle x\rangle^{2 \gamma-2}\right)
$$

for all $x$. Combining (3.33) with (2.4) we conclude that for any $\delta \in(0,1)$ there exists $X$, depending on parameters $n, \delta, k, \gamma$, such that

$$
\sum_{y=x-c_{n}\langle x\rangle^{\gamma}}^{x+c_{n}\langle x\rangle^{\gamma}}\left|a_{x y}\right|\left|\frac{\langle y\rangle^{k}}{\langle x\rangle^{k}}-1\right|<\frac{\delta}{\left(c_{n}+1\right)^{k}}, \quad|x| \geq \bar{X} .
$$

Using the above and (3.32) we get

$$
I_{1} \leq \frac{\delta}{2}\left\|T \tilde{f}^{(N)}\right\|_{\ell_{2}(\mathbb{Z})}^{2}
$$

Likewise, from the second equality in (3.30) and (3.26),

$$
I_{2} \leq \frac{1}{2} \sum_{y=\bar{X}}^{\bar{Y}} t_{y}^{2}\left|\tilde{f}_{y}^{(N)}\right|^{2} \sum_{x=y-c_{n}\langle y\rangle^{\gamma}}^{y+c_{n}\langle y\rangle^{\gamma}} \frac{t_{x}}{t_{y}^{2}}\left|a_{x y}\right|\left|\langle x\rangle^{k}-\langle y\rangle^{k}\right| .
$$

Since

$$
\frac{1}{C_{*}} \leq \frac{t_{x}}{\langle x\rangle^{k}} \leq C_{*}, \quad \bar{X}-c_{n}\langle\bar{X}\rangle^{\gamma} \leq x \leq \bar{Y}+c_{n}\langle\bar{Y}\rangle^{\gamma}
$$


for some constant $C_{*}>1$ that depends only on $n, \gamma, k$, we conclude that

$$
I_{2} \leq \frac{C_{*}^{3}}{2} \sum_{y=\bar{X}}^{\bar{Y}} t_{y}^{2}\left|\tilde{f}_{y}^{(N)}\right|^{2} \sum_{x=y-c_{n}\langle y\rangle^{\gamma}}^{y+c_{n}\langle y\rangle^{\gamma}}\left|a_{x y}\right| \frac{\langle x\rangle^{k}}{\langle y\rangle^{k}}\left|\frac{\langle x\rangle^{k}}{\langle y\rangle^{k}}-1\right| .
$$

Choose $X$ sufficiently large so that

$$
\sum_{x=y-c_{n}\langle y\rangle^{\gamma}}^{y+c_{n}\langle y\rangle^{\gamma}}\left|a_{x y}\right| \frac{\langle x\rangle^{k}}{\langle y\rangle^{k}}\left|\frac{\langle x\rangle^{k}}{\langle y\rangle^{k}}-1\right|<\frac{\delta}{C_{*}^{3}}, \quad|y| \geq \bar{X} .
$$

As a result we conclude

$$
I_{2} \leq \frac{\delta}{2}\left\|T \tilde{f}^{(N)}\right\|_{\ell_{2}(\mathbb{Z})}^{2}
$$

Combining this with (3.35) we have

$$
\left|\left\langle T \tilde{f}^{(N)},\left[T, A^{(N)}\right] \tilde{f}^{(N)}\right\rangle_{\ell_{2}(\mathbb{Z})}\right| \leq \delta \sum_{x} t_{x}^{2}\left|\tilde{f}_{x}^{(N)}\right|^{2}=\delta\left\|T \tilde{f}^{(N)}\right\|_{\ell_{2}}^{2} .
$$

Going back to (3.28) we obtain

$$
\begin{aligned}
\left\|T \tilde{f}^{(N)}\right\|_{\ell_{2}(\mathbb{Z})}^{2} & \leq\left|\left\langle T \tilde{f}^{(N)}, T g\right\rangle_{\ell_{2}(\mathbb{Z})}\right|+\left|\left\langle T \tilde{f}^{(N)},\left[T, A^{(N)}\right] \tilde{f}^{(N)}\right\rangle_{\ell_{2}(\mathbb{Z})}\right| \\
& \leq\left|\left\langle T \tilde{f}^{(N)}, T g\right\rangle_{\ell_{2}(\mathbb{Z})}\right|+\delta\left\|T \tilde{f}^{(N)}\right\|_{\ell_{2}(\mathbb{Z})}^{2},
\end{aligned}
$$

therefore

$$
\left\|T \tilde{f}^{(N)}\right\|_{\ell_{2}(\mathbb{Z})} \leq \frac{\|T g\|_{\ell_{2}(\mathbb{Z})}}{1-\delta}
$$

Now, we let the parameter $Y$, appearing in the definition of the operator $T$, tend to infinity. Since $\|T g\|_{\ell_{2}(\mathbb{Z})}$ remains constant, starting with some sufficiently large $Y$ (as $g \in c_{0}(\mathbb{Z})$ ) we infer that (3.39) implies (3.17). This ends the proof of (3.2) finishing also the proof of Theorem 2.1.

\section{Proof of Theorem 2.3}

Assume that $n$ is as in (1.3). We let

$$
\Gamma_{*}:=\sup _{y \in \mathbb{Z}} \frac{\langle y+2 n\rangle}{\langle y\rangle}
$$

and

$$
\hat{C}:=\sup _{x, y \in \mathbb{Z},|x-y| \leq n}\langle y\rangle\left|\frac{\langle x\rangle^{2}}{\langle y\rangle^{2}}-1\right| .
$$

One can easily see that $\Gamma_{*} \leq 2 n+1$ and $\hat{C} \leq n^{2}+2 n$. Suppose that $c_{*}>0$ appearing in (2.6) satisfies

$$
c_{*} \hat{C} \Gamma_{*}^{2}\left(1+\Gamma_{*}^{6}\right)<2 .
$$

The argument used in the proof of Theorem 2.1 can be easily adapted to the case when hypotheses (1.3) and (2.6) hold, provided that we are able to show the following modification of Proposition 3.1. 
Proposition 4.1. Assume that conditions (1.3) and (2.6) hold, with $c_{*}$ satisfying (4.3). Then, there exists $k_{0}>2$ such that for any $g \in c_{0}(\mathbb{Z})$ one can find $f=\left(f_{x}\right)$ satisfying $(3.2)$ and such that

$$
\sum_{x}\langle x\rangle^{2 k_{0}}\left|f_{x}\right|^{2}<+\infty .
$$

Proof. The proof of the proposition follows (with rather minor modifications) the argument used in the proof of Proposition 3.1. In place of (3.36) we can write, thanks to (4.1),

$$
\frac{1}{\Gamma_{*}^{k_{0}}} \leq \frac{t_{x}}{\langle x\rangle^{k}} \leq \Gamma_{*}^{k_{0}}, \quad X-2 n \leq x \leq Y+2 n,
$$

where $k_{0}>2$ and $0 \leq X \leq Y-2 n$ are to be adjusted later on. We invoke here an elementary bound, that is a consequence of convexity of the function $\phi(s):=s^{k_{0} / 2}$ : for any $M>1$ we have

$$
\left|s^{k_{0} / 2}-1\right| \leq \frac{k_{0}}{2} M^{k_{0} / 2-1}|s-1|, \quad 0 \leq s \leq M .
$$

From (4.2) and (4.6) we conclude that, cf. (3.33),

$$
\left|\frac{\langle y\rangle^{k_{0}}}{\langle x\rangle^{k_{0}}}-1\right| \leq \frac{k_{0} \hat{C} \Gamma_{*}^{k_{0}-2}}{2\langle x\rangle}, \quad|y-x| \leq n .
$$

Using the above together with (2.4) we conclude that for any $\epsilon>0$ there exists $X>0$ (appearing in the definition of the operator $T$ ), such that

$$
\sum_{y=x-n}^{x+n}\left|a_{x y}\right|\left|\frac{\langle y\rangle^{k_{0}}}{\langle x\rangle^{k_{0}}}-1\right| \leq \frac{1}{2}\left(c_{*}+\epsilon\right) k_{0} \hat{C} \Gamma_{*}^{k_{0}-2}, \quad|x| \geq X
$$

and, invoking also (4.1),

$$
\sum_{x=y-n}^{y+n}\left|a_{x y}\right| \frac{\langle x\rangle^{k_{0}}}{\langle y\rangle^{k_{0}}}\left|\frac{\langle x\rangle^{k_{0}}}{\langle y\rangle^{k_{0}}}-1\right| \leq \frac{1}{2}\left(c_{*}+\epsilon\right) k_{0} \hat{C} \Gamma_{*}^{2 k_{0}-2}, \quad|y| \geq X .
$$

Maintaining the notation from (3.30) we obtain the estimate [cf. (3.31) and (3.37)]

$$
I_{1}+I_{2} \leq \frac{1}{4}\left(c_{*}+\epsilon\right) k_{0} \hat{C} \Gamma_{*}^{2 k_{0}-2}\left(1+\Gamma_{*}^{3 k_{0}}\right)\left\|T \tilde{f}^{(N)}\right\|_{\ell_{2}(\mathbb{Z})}^{2}
$$

Choosing $k_{0}>2$ and $\epsilon>0$ in such a way that [cf. (4.3)]

$$
\delta:=\frac{k_{0}}{4}\left(c_{*}+\epsilon\right) \hat{C} \Gamma_{*}^{2 k_{0}-2}\left(1+\Gamma_{*}^{3 k_{0}}\right)<1
$$

we conclude that (3.39) is still in force. This ends the proof of the proposition 3.1 . 


\section{Acknowledgments}

The author wishes to express his gratitude to prof. J. Janas for bringing to his attention Ref. [3].

Open Access. This article is distributed under the terms of the Creative Commons Attribution 4.0 International License (http://creativecommons.org/licenses/ by/4.0/), which permits unrestricted use, distribution, and reproduction in any medium, provided you give appropriate credit to the original author(s) and the source, provide a link to the Creative Commons license, and indicate if changes were made.

\section{References}

[1] Akhiezer, N.I., Glazman, I.M.: Theory of Linear Operators in Hilbert Space. Dover, New York (1996)

[2] Edwards, R.E.: Functional Analysis. Theory and Applications. Holt, Rinehart (1965)

[3] Janas, J., Stochel, J.: Self-adjoint operator matrices with finite rows. Ann. Polon. Math. 66, 155-172 (1997)

[4] Kato, T.: Perturbation theory for linear operators. In: Reprint of the 1980 edition. Classics in Mathematics. Springer, Berlin (1995)

[5] Komorowski, T., Landim, C., Olla, S.: Fluctuations in Markov processes. In: Time Symmetry and Martingale Approximation. Grundlehren der Math. Wiss., vol. 345. Springer, Heidelberg (2012)

[6] Kostyuchenko, A.G., Mirzoev, K.A.: Complete indefiniteness tests for Jacobi matrices with matrix entries. Funct. Anal. Appl. 35(4), 265-269 (2001)

[7] Krein, M.G.: Infinite J-matrices and the matrix moment problem. Dokl. Akad. Nauk SSSR 69(3), 125-128 (1949)

[8] Krein, M.G.: Basic statements of the theory of representations of Hermitian operators with deficiency indices (m,m). Ukr. Matem. Zh. 2, 366 (1949)

[9] Kogan, V.I.: On the operators generated by lp matrices with maximal deficiency indices. Teoriya Funktsii, Funkts. Anal. i Prilozhen. (Kharkov) 11, 103$107(1970)$

[10] Lax, P.: Functional Analysis. Wiley, New York (2002)

[11] Nagy, B.: Multiplicities, generalized Jacobi matrices, and symmetric operators. J. Oper. Theory 65(1), 211-232 (2011)

[12] Sethuraman, S., Varadhan, S.R.S., Yau, H.-T.: Diffusive limit of a tagged particle in asymmetric simple exclusion processes. Commun. Pure Appl. Math. 53(8), 972-1006 (2000)

[13] Simon, B.: The classical moment problem as a self-adjoint finite difference operator. Adv. Math. 137, 82-203 (1998)

[14] Smart, D.R.: Representation of Hilbert space operators by $(n J)$-matrices. Math. Proc. Camb. Phil. Soc. 53 1, 304-311 (1957) 
Tomasz Komorowski $(\bowtie)$

Institute of Mathematics, Polish Academy of Sciences,

Śniadeckich 8, 00-656, Warsaw,

Poland

e-mail: komorow@hektor.umcs.lublin.pl

Received: October 11, 2014.

Revised: April 27, 2015. 